\title{
Surgical Approach to Control Advanced Coats Disease
}

\author{
Şengül Özdek ${ }^{1}$, Ahmet Yücel Üçgül ${ }^{2}$ \\ ${ }^{1}$ Professor in Ophthalmology, Gazi University, School of Medicine, Ankara \\ ${ }^{2}$ Abantlzzet Baysal University Training and Research Hospital, Bolu, Turkey
}

Coats disease, first described by George Coats in 1908 , is commonly a unilateral clinical entity characterized by leaky telangiectatic vessels leading to progressive subretinal exudation and exudative retinal detachment (ERD). ${ }^{1}$ It is generally seen in pediatric population and has a distinct male predilection of over $90 \%$. Shields et al. classified Coats disease into five stages as follows: Stage 1: presence of retinal telangiectasia only, Stage 2: telangiectasia and exudation (2A: extrafoveal exudation, 2B: foveal exudation), Stage 3: exudative RD (3A: subtotal RD, 3B: total RD), Stage 4: total RD and neovascular glaucoma, Stage 5: advanced end-stage disease. ${ }^{2}$

Disease progression can be controlled with ablative therapies such as laser photocoagulation (LFK) and cryotherapy in the early stages of Coats disease. However, ablative therapies are not effective in the late stages (Stage $3-4$ ) due to excessive subretinal exudation and ERD. Therefore, adjunctive surgical approaches have become popular in the treatment of advanced Coats disease. This helps in effective ablation of leaky abnormal vessels.

In the presence of ERD, although some authors are of the opinion that laser energy could be absorbed by haemoglobin in the telangiectetic vessels on the

How to Cite this Article: Özdek Ş, Üçgül AY. Surgical Approach to Control Advanced Coats Disease. Pak J Ophthalmol. 2020; 36 (4): 456-458.

Doi: https://doi.org/10.36351/pjo.v36i4.1112

Correspondence: Şengül Özdek

Gazi University, School of Medicine, Ankara, Turkey

E mail: sengulozdek@gmail.com

Received: June 20, 2020

Accepted: July 29, 2020 detached retina. ${ }^{3}$ However, we consider that external drainage of subretinal fluid and exudation is necessary because ERD can obscure the visualization of the peripheral part of the retina. Furthermore, the ablative therapies can be applied more effectivelywhen retina is attached, as Retinal pigment epithelium (RPE) contains melanin which has a stronger ability of absorption of laser energy when compared to haemoglobin. Transscleral drainage of subretinal fluid (TDSRF) enhances the visualization of the retina and contributes to more effective ablation by providing retinal reattachment prior to ablative therapies. Another advantage of this surgical approach is the maintenance of retinal integrity (Figure 1). Up till now, favourable anatomic outcomes have been reported after the TDSRF, which was performed as an adjunct to ablative therapies. However, visual outcomes remained poor due to the presence of severe photoreceptor damage in advanced cases. ${ }^{4}$

In the last decade, satisfactory outcomes have been reported after pars plana vitrectomy (PPV) surgery, which was performed to treat advanced Coats disease. ${ }^{5}$ The first purpose of PPV surgery is the removal of vascular endothelial growth factor (VEGF) burden, which increases secondary to the retinal ischemia. The

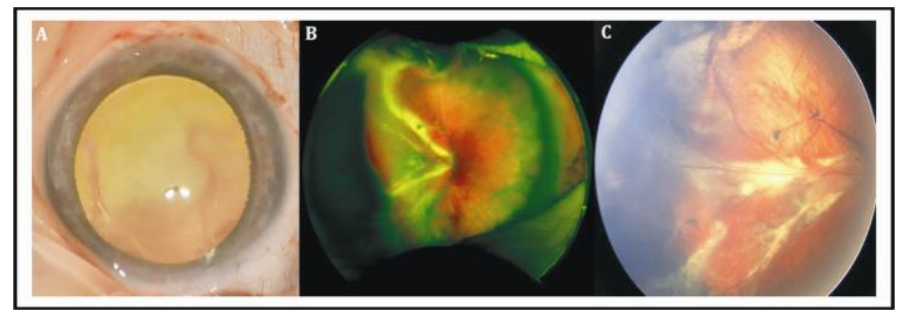

Fig. 1: Preoperative image of a 9-month-old baby having unilateral stage $3 b$ Coats-related exudative RD (A). External drainage together with cryotherapy combined with anti-VEGF injection at the end of the surgery resulted in complete reattachment of the retina at one month (B) and 12 months follow-up visits (C). 
second aim of PPV is peeling of the posterior hyaloid membrane, which acts as a scaffold for potential subsequent PVR. Although some surgeons performed an internal drainage retinotomy to drain the subretinal fluid and exudate and reported relatively successful outcomes after the surgery, 5,6 we consider that internal retinotomy can predispose to the development of proliferative vitreoretinopathy (PVR) and rhegmatogenous retinal detachment (RRD) in such an aggressive disease with excessive exudations. Therefore, because the maintenance of the retinal integrity is crucial in advanced coats disease, we prefer to avoid internal drainage (Figure 2).

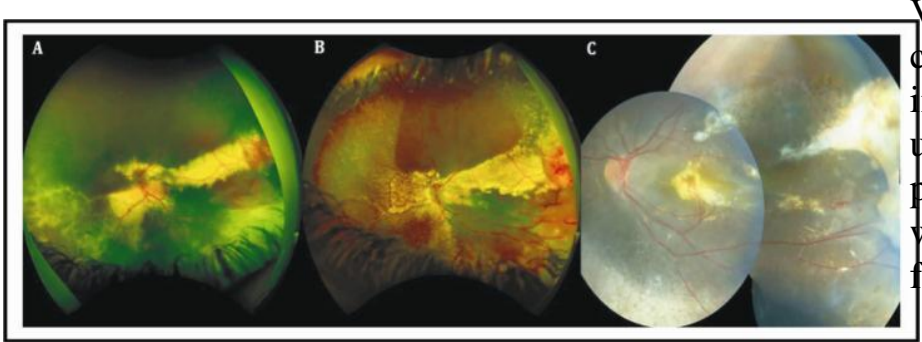

TRD has a limited contribution to visual outcomes but it can slow the disease progression and reduce the need for further ablative therapy. Therefore, we recommend PPV together with removal of posterior hyaloid when possible and other tractional membranes in advanced cases with TRD.

Another important question is that; should PPV be combined with external drainage in advanced disease or it should be reserved when further TRD develops? TDSRF is accepted by many surgeons for effective ablation of the abnormal leaky vessels in advanced Coats disease. Some cases, where TDSRF was performed as an adjunct to ablative therapies, developed TRD secondary to cryotherapy and antiVEGF therapyin the long-term follow-up and these qases were treated with PPV. ${ }^{10}$ Our 10-year experience in the treatment of advanced Coats disease has shown is that simultaneous surgeries of TDSRF and PPV protects from further development of TRD. In cases, where TDSRF and PPV are performed, the need for further ablative therapy decreases (unpublished data). ${ }^{11}$

Fig. 2: Preoperative image of 12-year-old boy with stage 3 a Coats (A). There was limited subretinal fluid (no bullous RD) with subretinal hard exudates, old vitreous haemorrhage opacity in inferior vitreous cavity and epiretinal membranes. PPV with endolaser and cryotherapy to the temporal quadrants and Anti-VEGF injection without external subretinal fluid drainage was performed. Retina was reattached with less subretinal exudates during the $1^{\text {st }}$ month visit (B) and there was subretinal fibrosis in the macula and temporal periphery with totally attached retina without significant exudate at the last visit, which was $12^{\text {th }}$ postoperative month (C).

Vitreoretinal fibrosis and Tractional retinal detachment (TRD) is not seen in the natural clinical course of Coats disease. ${ }^{7}$ It is accepted that TRD develops secondary to the ablative therapies. In a large series of 351 cases, in which the potential risk factors for the development of TRD were investigated, it was found that cryotherapy and anti-VEGF therapy increased the risk of TRD development in Coats disease. ${ }^{8}$ This poses two questions: Does TRD worsen the clinical course of advanced Coats disease? Does treatment of TRD contributes positively to the clinical course of advanced Coats Disease?

It is known that visual outcomes are poor due to severe photoreceptor damage secondary to excessive sub-foveal exudation in advanced Coats disease. Some authors consider that the clinical approach should be limited to observation in such cases with TRD. ${ }^{9}$ However, in advanced cases, although treatment of

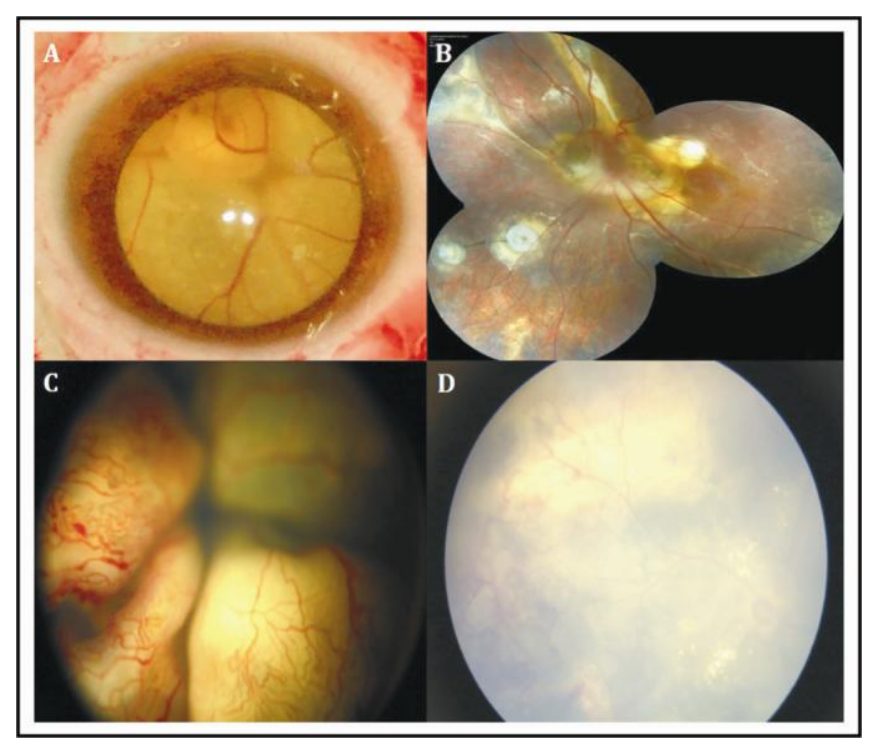

Fig. 3: Preoperative image of 6-year-old girl, which shows total exudative retinal detachment in contact with the lens $(\mathbf{A})$. External drainage together with PPV, endolaser \& cryotherapy to telangiectasic areas combined with antiVEGF injection at the end of the surgery resulted in complete reattachment of the retina at 18 months follow-up visits. At last visit, retina seems to be attached completely, together with widespread subretinal fibrosis and subfoveal nodule (B). Another preoperative image of 2-year-old girl, which shows total exudative retinal detachment and widespread abnormal telangiectatic vessel (C). The patient had an operation by the same surgical technique. At first year of the follow-up, retina seems to be attached completely, and ablated abnormal telangiectatic vessels seems to be under control without leaking (D). 
When TDSRF is performed alone, sub-retinal exudation may recur in the long term and ERD may occur together with TRD. Consequently, although the first surgery is more invasive, there will be no need for a second surgery as the risk of subsequent TRD development is eliminated in patients undergoing simultaneous surgeries of TDSRF and PPV (Figure 3).

In early stages (stage $1-2$ ) of Coats disease, effective ablation of leaky vessels could be achieved with application of laser photocoagulation and cryotherapy when necessary. Furthermore, the use of anti-VEGF agents also contributes to a decrease in macular oedema and subretinal fluid. However, patients with advanced Coats disease require more than conventional ablative therapies. Adjunctive surgical approaches include TDSRF and PPV. Simultaneous surgeries of PPV and TDSRF are more successful, when compared with sequential surgeries. Internal drainage should be avoided to decrease the risk of PVR and RRD development at a later date.

\section{Conflict of Interest}

Authors declared no conflict of interest.

\section{REFERENCES}

1. Coats G. Forms of retinal diseases with massive exudation. Roy Lond Ophthalmol Hosp Rep. 1908; 17: 440-525.

2. Shields JA, Shields CL, Honavar SG, Demirci H, Cater J. Classification and management of Coats disease: the 2000 Proctor Lecture. Am J Ophthalmol. 2001; 131: 572-583.

3. Nucci P, Bandello F, Serafino M, Wilson ME. Selective photocoagulation in Coats' disease: ten-year follow-up. Eur J Ophthalmol. 2002; 12 (6): 501-505.
4. Peng J, Zhang Q, Jin H, Fei P, Zhao P. A Modified Technique for the Transconjunctival and Sutureless External Drainage of Subretinal Fluid in Bullous Exudative Retinal Detachment Using a 24-G i.v. Catheter. Ophthalmologica. 2017; 238 (4): 179-185.

5. Karacorlu M, Hocaoglu M, Sayman Muslubas I, Arf S. Long-Term Anatomical and Functional Outcomes Following Vitrectomy for Advanced Coats Disease. Retina, 2017; 37 (9): 1757-1764.

6. Muftuoglu G, Gulkilik G. Pars plana vitrectomy in advanced coats' disease. Case Rep Ophthalmol. 2011; 2 (1): $15-22$.

7. Shields JA, Shields CL, Honavar SG, Demirci H. Clinical variations and complications of Coats disease in 150 cases: the 2000 Sanford Gifford Memorial Lecture. Am J Ophthalmol. 2001; 131 (5): 561-571.

8. Shields CL, Udyaver S, Dalvin LA, Lim LAS, Atalay HT, Khoo CT, Shields JA. Coats disease in 351 eyes: Analysis of features and outcomes over 45 years (by decade) at a single center. Ind J Ophthalmol. 2019; 67 (6): 772 .

9. Adeniran, Janelle Fassbender, Nathan Lambert N, Ramasubramanian A. "Treatment of Coats' disease: an analysis of pooled results." Int J Ophthalmol. 2019; 12 (4): 668.

10. Li AS, Capone A, Jr., Trese MT, et al. Long-Term Outcomes of Total Exudative Retinal Detachments in Stage 3B Coats Disease. Ophthalmology, 2018; 125 (6): 887-893.

11. Ucgul AY, Ozdek S, Ertop M, Atalay HT. Surgical Approaches as an adjunct to ablative therapy in advanced Coats disease. TOA $53^{\text {rd }}$ National Congress. 2019; Oral Presentation.

\section{Authors' Designation and Contribution}

Şengül Özdek: Professor; Study Design, Manuscript preparation, final review.

Ahmet Yücel Üçgül: Study Design, Manuscript preparation, final review. 